\title{
Periodic Solutions of a Stage-Structured Plant-Hare Model with Toxin-Determined Functional Responses
}

\author{
Huicheng Wang, ${ }^{1}$ Patricia J. Y. Wong, ${ }^{2}$ and Yonghui Xia ${ }^{1}$ \\ ${ }^{1}$ Department of Mathematics, Zhejiang Normal University, Jinhua, Zhejiang 321004, China \\ ${ }^{2}$ School of Electrical and Electronic Engineering, Nanyang Technological University, Singapore 639798 \\ Correspondence should be addressed to Yonghui Xia; yhxia@zjnu.cn
}

Received 8 April 2014; Revised 25 April 2014; Accepted 26 April 2014; Published 8 May 2014

Academic Editor: Tonghua Zhang

Copyright (c) 2014 Huicheng Wang et al. This is an open access article distributed under the Creative Commons Attribution License, which permits unrestricted use, distribution, and reproduction in any medium, provided the original work is properly cited.

The purpose of this paper is to obtain some sufficient conditions for the global existence of multiple positive periodic solutions of a delayed stage-structured plant-hare model with a toxin-determined functional response. Some novel estimation techniques to construct two open subsets for a priori bounds are employed.

\section{Introduction}

A lot of classical predator-prey models have been well studied (e.g., see [1-12]). Recently, Gao et al. [13] considered a nonautonomous plant-hare dynamical system with a toxindetermined functional response given by

$$
\begin{aligned}
\dot{N}(t) & =r(t) N(t)\left[1-\frac{N(t)}{K}\right]-C(N(t)) P(t), \\
\dot{P}(t) & =B(t) C(N(t)) P(t)-d(t) P(t), \\
C(N(t)) & =f(N(t))\left[1-\frac{f(N(t))}{4 G}\right], \\
f(N(t)) & =\frac{e \delta N(t)}{1+h e \delta N(t)},
\end{aligned}
$$

where $N(t)$ denotes the density of plant at time $t$ and $P(t)$ denotes the herbivore biomass at time $t$.

On the other hand, many experts argued that the predator-prey models should be modified to fit the more realistic environment. They suggested that one should take the stage structure factor into consideration. Because it is very unrealistic to assume that each individual predator admits the same ability of attacking in the classical predator-prey models. They divided the individuals into two stages in life history, namely, immature and mature stages, where the rate of the immature predator attacking the prey and the reproductive rate can be ignored, while the mature predators are responsible for the prey. For example, one can refer to $[14,15]$ and the references cited therein. To discuss the effects of Holling type IV functional responses on a stage-structured model, the authors in [16] proposed the following delayed system:

$$
\begin{aligned}
\frac{d x(t)}{d t}= & x(t)\left[r_{1}(t)-a_{1}(t)\right. \\
& \times \int_{-\infty}^{t} K(t-s) x(s) d s \\
& \left.-\frac{a_{2}(t) y_{2}(t)}{x^{2}(t) / m+x(t)+a}\right], \\
\frac{d y_{1}(t)}{d t}= & \frac{b_{1}(t) x(t) y_{2}(t)}{x^{2}(t) / m+x(t)+a}-\beta(t) y_{1}(t) \\
& -b_{1}(t-\tau) \exp \left(-\int_{t-\tau}^{t} \beta(s) d s\right) \\
& \times \frac{x(t-\tau) y_{2}(t-\tau)}{x^{2}(t-\tau) / m+x(t-\tau)+a}, \\
\frac{d y_{2}(t)}{d t}= & b_{1}(t-\tau) \exp \left(-\int_{t-\tau}^{t} \beta(s) d s\right) \\
& \times \frac{x(t-\tau) y_{2}(t-\tau)}{x^{2}(t-\tau) / m+x(t-\tau)+a} \\
& -r_{2}(t) y_{2}(t) .
\end{aligned}
$$


However, Holling IV type functional response is not appropriate for the plant-hare model if we explore the impact of plant toxicity on the dynamics of plant-hare interactions. Because such kind of plant can produce toxicity to protect itself. Therefore, in the present paper, we discuss the stage-structured plant-hare model with toxin-determined functional response as follows:

$$
\begin{aligned}
& \frac{d x(t)}{d t}=x(t)\left[r_{1}(t)-a(t) x(t)\right. \\
& \left.-\frac{4 G e \delta y_{2}(t)+(4 G h-1) e^{2} \delta^{2} x(t) y_{2}(t)}{4 G(1+h e \delta x(t))^{2}}\right] \\
& \frac{d y_{1}(t)}{d t}=-\beta(t) y_{1}(t) \\
& +\left(\left(b_{1}(t) 4 G e \delta B x(t) y_{2}(t)\right.\right. \\
& \left.+(4 G h-1) e^{2} \delta^{2} B x^{2}(t) y_{2}(t)\right) \\
& \left.\times\left(4 G(1+h e \delta x(t))^{2}\right)^{-1}\right) \\
& -b_{1}(t-\tau) \exp \left\{-\int_{t-\tau}^{t} \beta(s) d s\right\} \\
& \times\left(\left(4 G e \delta B x(t-\tau) y_{2}(t-\tau)\right.\right. \\
& \left.+(4 G h-1) e^{2} \delta^{2} B x^{2}(t-\tau) y_{2}(t-\tau)\right) \\
& \left.\times\left(4 G(1+h e \delta x(t-\tau))^{2}\right)^{-1}\right), \\
& \frac{d y_{2}(t)}{d t}=b_{1}(t-\tau) \exp \left\{-\int_{t-\tau}^{t} \beta(s) d s\right\} \\
& \times\left(\left(4 G e \delta B x(t-\tau) y_{2}(t-\tau)\right.\right. \\
& \left.+(4 G h-1) e^{2} \delta^{2} B x^{2}(t-\tau) y_{2}(t-\tau)\right) \\
& \left.\times\left(4 G(1+h e \delta x(t-\tau))^{2}\right)^{-1}\right) \\
& -r_{2}(t) y_{2}(t)
\end{aligned}
$$

where $x(t)$ denotes the density of the plant at time $t, y_{1}(t)$ is the density of immature individual hares at time $t$, and $y_{2}(t)$ denotes the density of mature individual hares at time $t$, respectively; $r_{1}(t), r_{2}(t), a(t), b_{1}(t)$, and $\beta(t)$ are continuously positive periodic functions with period $\omega . B$ is the conversion rate, $e$ is the encounter rate per hare, $\delta$ is the fraction of food items encountered that the hares ingest, $G$ measures the toxicity level, and $h$ is the time for handing one unit of plant. $e, \delta, G$, and $h$ are positive real constants. $r_{1}(t)$ is the intrinsic growth rate of the prey, $a(t)$ is the density-dependent coefficient of the plant, and $r_{2}(t)$ is the death rate of the mature hares.
For any continuous $\omega$-periodic function $f(t)$, we always adopt the following notations throughout this paper:

$$
\begin{gathered}
\bar{f}=\frac{1}{\omega} \int_{0}^{\omega} f(t) d t, \quad f^{L}=\min _{t \in[0, \omega]} f(t), \\
f^{M}=\max _{[0, \omega]} f(t),
\end{gathered}
$$

where $f$ is a continuous $\omega$-periodic function.

The purpose of this paper is to obtain some sufficient conditions for the global existence of multiple positive periodic solutions of system (4). Our method is based on Mawhin's coincidence degree and novel estimation techniques for a priori bounds of unknown solutions to $L x=\lambda N x$. To the best of our knowledge, it is the first time that a delayed stage-structured plant-hare dynamical system with a toxindetermined functional response has been proposed and studied by using this method.

Remark 1. The term $\exp \left\{-\int_{t-\tau}^{t} \beta(s) d s\right\}\left(x(t-\tau) y_{2}(t-\tau) /\left(x^{2}(t-\right.\right.$ $\tau) / m+x(t-\tau)+a))$ in the third equation of (4) involves $x(t-\tau) y_{2}(t-\tau)$ instead of $x(t-\tau) y_{2}(t)$; the method used in [13] cannot be applied to system (4) directly. Thus, novel estimation techniques must be employed for a priori bounds of unknown solutions to the operator equation $L x=\lambda N x$. More specifically, integrating the second equation of system (1) over $[0, \omega]$, the authors in [13] obtained

$$
\begin{aligned}
& \int_{0}^{\omega}(\left(4 G e \delta B(t) \exp \left\{u_{1}(t)\right\}\right. \\
&\left.+(4 G h-1) e^{2} \delta^{2} B(t) \exp \left\{2 u_{1}(t)\right\}\right) \\
&\left.\quad \times\left(4 G\left(1+h e \delta \exp \left\{u_{1}(t)\right\}\right)^{2}\right)^{-1}\right) d t \\
&=\bar{d} \omega .
\end{aligned}
$$

It follows that

$$
\bar{d} \omega \geq \int_{0}^{\omega} \frac{4 G e \delta B(t) \exp \left\{u_{1}(t)\right\}}{4 G\left(1+h e \delta \exp \left\{u_{1}(t)\right\}\right)^{2}} d t .
$$

By some arguments, this inequality then leads them to

$$
\begin{aligned}
& \bar{d} h^{2} e^{2} \delta^{2} \exp \left(2 u_{1}\left(\eta_{1}\right)\right) \\
& -(e \delta \bar{B} \exp \{-2 \bar{r} \omega\}-2 h e \delta \bar{d}) \\
& \times \exp \left\{u_{1}\left(\eta_{1}\right)\right\}+\bar{d}>0,
\end{aligned}
$$

which implies that

$$
x_{1}\left(\eta_{1}\right)<\ln h_{-}^{0}, \quad x_{1}\left(\eta_{1}\right)>\ln h_{+}^{0},
$$

where

$$
\begin{aligned}
h_{ \pm}^{0} & =\frac{(e \delta \bar{B} \exp \{-2 \bar{r} \omega\}-2 h e \delta \bar{d}) \pm \sqrt{\Delta_{1}}}{2 \bar{d} h^{2} e^{2} \delta^{2}} \\
\Delta_{1} & =[e \delta \bar{B} \exp \{-2 \bar{r} \omega\}-2 h e \delta \bar{d}]^{2}-4 \bar{d}^{2} h^{2} e^{2} \delta^{2}
\end{aligned}
$$


It should be noted that it is possible to construct two open subsets $\Omega_{1}$ and $\Omega_{2}$ due to (9). The essential reason to obtain (9) is the inequality (7). In inequality (7), there is no variable $u_{2}(t)$ and only one variable $u_{1}(t)$.

However, since the term $x(t-\tau) y_{2}(t-\tau)$ is in the third equation of (4), by same arguments in [13], we will see that

$$
\begin{aligned}
& \int_{0}^{\omega} b_{1}(t-\tau) \exp \left\{-\int_{t-\tau}^{t} \beta(s) d s\right\} \\
& \quad \times \exp \left\{u_{2}(t-\tau)-u_{2}(t)\right\} \\
& \times\left(\left(4 G e \delta B \exp \left\{u_{1}(t-\tau)\right\}\right.\right. \\
& \left.\quad+(4 G h-1) e^{2} \delta^{2} B \exp \left\{2 u_{1}(t-\tau)\right\}\right) \\
& \left.\quad \times\left(4 G\left(1+h e \delta \exp \left\{u_{1}(t-\tau)\right\}\right)^{2}\right)^{-1}\right) d t \\
& =\bar{r}_{2} \omega .
\end{aligned}
$$

Note that both $u_{1}$ and $u_{2}$ appear simultaneously in the above equality. If we were to use the same ideas in [13], then the above equality does not lead us anywhere. Thus, some new arguments should be employed to obtain a priori bounds for $u_{1}$. To see how to overcome this difficulty, the reader can refer to (33)-(56) in Section 2.

Remark 2. It should be noted that the standard estimation techniques used in [16] are not applicable to the system (4) either, due to the term $C(N(t))$. If we were to use the standard arguments in [16], we can not obtain two positive roots of $\exp \left(u_{1}\left(\xi_{1}\right)\right)$. Consequently, we can not construct two open subsets. Thus, we can not obtain two positive solutions in these two open subsets.

\section{Existence of Multiple Positive Periodic Solutions}

In this section, we will study the existence of multiple periodic solutions of (4). We recall a few concepts and results from [17].

Lemma 3 (see [17]). Let $\Omega \subset X$ be an open bounded set. Let $L$ be a Fredholm mapping of index zero and NL-compact on $\bar{\Omega}$. Assume

(a) for each $\lambda \in(0,1), x \in \partial \Omega \cap \operatorname{Dom} L, L x \neq \lambda N x$;

(b) for each $x \in \partial \Omega \cap \operatorname{Ker} L, Q N x \neq 0$;

(c) $\operatorname{deg}\{J Q N, \Omega \cap \operatorname{Ker} L, 0\} \neq 0$.

Then $L x=N x$ has at least one solution in $\bar{\Omega} \cap \operatorname{Dom} L$.

Lemma 4. If $\beta(t)$ and $g(t)$ are $\omega$-periodic functions, then the system

$$
\frac{d y(t)}{d t}=\beta(t) y(t)+g(t)
$$

has a unique $\omega$-periodic solution which can be represented as $y(t)=\int_{-\infty}^{t} \exp \left(\int_{s}^{t} \beta(\sigma) d \sigma\right) g(s) d s$
Throughout, we assume the following:

$$
\begin{aligned}
& \left(A_{1}\right) 1 / 4 h<G<1 / 3 h ; \\
& \left(A_{2}\right) 4 h r_{2}^{M} \exp \left\{\tau \beta^{M}\right\} \exp \left\{2 \bar{r}_{1} \omega\right\} / b_{1}^{L}<B<4 G r_{2}^{L} h^{2}\left(b_{1}^{M}\right)^{-1} \\
& \quad \exp \left\{\tau \beta^{L}\right\} /(4 G h-1) .
\end{aligned}
$$

We further introduce six positive numbers which will be used later as follows:

$$
\begin{aligned}
h_{ \pm}= & \left(\left(b_{1}^{L} e \delta B \exp \left\{-\tau \beta^{M}\right\} \exp \left\{-2 \bar{r}_{1} \omega\right\}-2 h e \delta r_{2}^{M}\right)\right. \\
& \left. \pm \sqrt{\Delta_{1}}\right) \times\left(2 r_{2}^{M} h^{2} e^{2} \delta^{2}\right)^{-1} \\
l_{ \pm}= & \left(\left[4 G h^{2} e \delta B \exp \left\{2 \bar{r}_{1} \omega\right\}\right.\right. \\
& \left.-2 h e \delta\left(4 G h^{2} r_{2}^{L}\left(b_{1}^{M}\right)^{-1} \exp \left\{\tau \beta^{L}\right\}-(4 G h-1) B\right)\right] \\
& \left. \pm \sqrt{\Delta_{2}}\right) \\
& \times\left(2 h ^ { 2 } e ^ { 2 } \delta ^ { 2 } \left[4 G h^{2} r_{2}^{L}\left(b_{1}^{M}\right)^{-1} \exp \left\{\tau \beta^{L}\right\}\right.\right. \\
u_{ \pm}= & \frac{\left(4 G e \delta B-8 G h e \delta \bar{r}_{2} \bar{b}^{-1}\right) \pm \sqrt{\Delta_{3}}}{2\left[4 G \bar{r}_{2} \bar{b}^{-1} h^{2} e^{2} \delta^{2}-(4 G h-1) e^{2} \delta^{2} B\right]},
\end{aligned}
$$

where

$$
\begin{aligned}
\Delta_{1}= & {\left[b_{1}^{L} e \delta B \exp \left\{-\tau \beta^{M}\right\} \exp \left\{-2 \bar{r}_{1} \omega\right\}-2 h e \delta r_{2}^{M}\right]^{2} } \\
& -4\left(r_{2}^{M}\right)^{2} h^{2} e^{2} \delta^{2}, \\
\Delta_{2}= & {\left[4 G h^{2} e \delta B \exp \left\{2 \bar{r}_{1} \omega\right\}\right.} \\
& \left.-2 h e \delta\left(4 G h^{2} r_{2}^{L}\left(b_{1}^{M}\right)^{-1} \exp \left\{\tau \beta^{L}\right\}-(4 G h-1) B\right)\right]^{2} \\
& -4 h^{2} e^{2} \delta^{2}\left[4 G h^{2} r_{2}^{L}\left(b_{1}^{M}\right)^{-1} \exp \left\{\tau \beta^{L}\right\}-(4 G h-1) B\right]^{2}, \\
\Delta_{3}= & \left.4 G e \delta B-8 G h e \delta \bar{r}_{2} \bar{b}^{-1}\right)^{2} \\
& -16 G \bar{r}_{2} \bar{b}^{-1}\left[4 G \bar{r}_{2} \bar{b}^{-1} h^{2} e^{2} \delta^{2}-(4 G h-1) e^{2} \delta^{2} B\right],
\end{aligned}
$$

$\bar{b}=(1 / \omega) \int_{0}^{\omega} b_{1}(t) \exp \left\{-\int_{t}^{t+\tau} \beta(s)\right\} d s d t$. Under assumptions $\left(A_{1}\right)$ and $\left(A_{2}\right)$, it is not difficult to show that

$$
l_{-}<u_{-}<h_{-}<h_{+}<u_{+}<l_{+} .
$$

Theorem 5. In addition to $\left(A_{1}\right)$ and $\left(A_{2}\right)$, suppose that

$$
\left(A_{3}\right) \bar{r}_{1}-\bar{a} \exp \left\{\ln l_{+}+2 \bar{r} \omega\right\}>0 .
$$

Then system (4) has at least two positive w-periodic solutions. 
Proof. Note that the first equation and the third equation of (4) can be separated from the whole system. Consider the following subsystem:

$$
\begin{aligned}
& \frac{d x(t)}{d t}=x(t)\left[r_{1}(t)-a(t) x(t)\right. \\
& \left.-\frac{4 G e \delta y_{2}(t)+(4 G h-1) e^{2} \delta^{2} x(t) y_{2}(t)}{4 G(1+h e \delta x(t))^{2}}\right], \\
& \frac{d y_{2}(t)}{d t}=b_{1}(t-\tau) \exp \left\{-\int_{t-\tau}^{t} \beta(s) d s\right\} \\
& \times\left(\left(4 G e \delta B x(t-\tau) y_{2}(t-\tau)+(4 G h-1) e^{2} \delta^{2}\right.\right. \\
& \left.\times B(t) x^{2}(t-\tau) y_{2}(t-\tau)\right) \\
& \left.\times\left(4 G(1+h e \delta x(t-\tau))^{2}\right)^{-1}\right) \\
& -r_{2}(t) y_{2}(t)
\end{aligned}
$$

Make the change of variables

$$
x(t)=\exp \left\{u_{1}(t)\right\}, \quad y(t)=\exp \left\{u_{2}(t)\right\} ;
$$

then system (16) can be rewritten as

$$
\begin{aligned}
\dot{u}_{1}(t)=r_{1}( & t)-a(t) \exp \left\{u_{1}(t)\right\} \\
- & \left(\left(4 G e \delta \exp \left\{u_{2}(t)\right\}+(4 G h-1) e^{2} \delta^{2}\right.\right. \\
& \left.\times \exp \left\{u_{1}(t)+u_{2}(t)\right\}\right) \\
& \left.\times\left(4 G\left(1+h e \delta \exp \left\{u_{1}(t)\right\}\right)^{2}\right)^{-1}\right):=f_{1}(t, u), \\
\dot{u}_{2}(t)=- & r_{2}(t)+b_{1}(t-\tau) \exp \left\{-\int_{t-\tau}^{t} \beta(s) d s\right\} \\
& \times( \\
& \quad \times \operatorname{ex} \delta B \exp \left\{u_{1}(t-\tau)\right\}+(4 G h-1) e^{2} \delta^{2} B \\
& \left.\times\left(4 G\left(1+h e \delta \exp \left\{u_{1}(t-\tau)\right\}\right)^{2}\right)^{-1}\right) \\
& \times \exp \left\{u_{2}(t-\tau)-u_{2}(t)\right\}:=f_{2}(t, u) .
\end{aligned}
$$

Take

$$
X=Y=\left\{x=\left(u_{1}, u_{2}\right)^{T} \in C\left(\mathbb{R}, \mathbb{R}^{2}\right) \mid x(t+\omega)=x(t)\right\}
$$

and define

$$
\begin{array}{r}
\|x\|=\max _{t \in[0, \omega]}\left|u_{1}(t)\right|+\max _{t \in[0, \omega]}\left|u_{2}(t)\right|, \\
x=\left(u_{1}, u_{2}\right)^{T} \in X \text { or } Y ;
\end{array}
$$

here $|\cdot|$ denotes the Euclidean norm. Then $X$ and $Y$ are Banach spaces with the norm $\|\cdot\|$. Set

\section{$L: \operatorname{Dom} L \cap X$,}

$$
L\left(u_{1}(t), u_{2}(t)\right)^{T}=\left(\frac{d u_{1}(t)}{d t}, \frac{d u_{2}(t)}{d t}\right)^{T}
$$

where Dom $L=\left\{\left(u_{1}(t), u_{2}(t)\right)^{T} \in C^{1}\left(\mathbb{R}, \mathbb{R}^{2}\right)\right\}$. Further, $N$ : $X \rightarrow X$ is defined by

$$
N\left(\begin{array}{l}
u_{1} \\
u_{2}
\end{array}\right)=\left(\begin{array}{l}
f_{1}(t, u) \\
f_{2}(t, u)
\end{array}\right)
$$

Define

$$
\begin{aligned}
P\left(\begin{array}{l}
u_{1} \\
u_{2}
\end{array}\right)=Q\left(\begin{array}{l}
u_{1} \\
u_{2}
\end{array}\right)= & \left(\begin{array}{l}
\frac{1}{\omega} \int_{0}^{\omega} u_{1}(t) d t \\
\frac{1}{\omega} \int_{0}^{\omega} u_{2}(t) d t
\end{array}\right), \\
& \left(\begin{array}{l}
u_{1} \\
u_{2}
\end{array}\right) \in X=Y .
\end{aligned}
$$

It is not difficult to show that $L$ is a Fredholm mapping of index zero. Furthermore, the generalized inverse (to $L$ ) $K_{p}$ : $\operatorname{Im} L \rightarrow$ Dom $L \cap$ Ker $P$ exists. Standard arguments show that $N$ is $L$-compact on $\bar{\Omega}$ for any open bounded set $\Omega \subset X$.

Now, we will search for two appropriate open bounded subsets in order to apply the continuation theorem.

Corresponding to the operator equation $L x=\lambda N x, \lambda \in$ $(0,1)$, we have

$$
\begin{aligned}
& \dot{u}_{1}(t)=\lambda r_{1}(t) \\
&-\lambda\left[a(t) \exp \left\{u_{1}(t)\right\}\right.-\left(\left(4 G e \delta \exp \left\{u_{2}(t)\right\}+(4 G h-1) e^{2} \delta^{2} \quad(24)\right.\right. \\
&\left.\times \exp \left\{u_{1}(t)+u_{2}(t)\right\}\right) \\
&\left.\left.\times\left(4 G\left(1+h e \delta \exp \left\{u_{1}(t)\right\}\right)^{2}\right)^{-1}\right)\right], \\
& \dot{u}_{2}(t)=-\lambda r_{2}(t) \\
&+\lambda\left[b_{1}(t-\tau) \exp \left\{-\int_{t-\tau}^{t} \beta(s) d s\right\}\right. \\
& \quad \times\left(\left(4 G e \delta B \exp \left\{u_{1}(t-\tau)\right\}+(4 G h-1) e^{2} \delta^{2} B\right.\right. \\
&\left.\quad \times \exp \left\{2 u_{1}(t-\tau)\right\}\right) \\
&\left.\quad \times\left(4 G\left(1+h e \delta \exp \left\{u_{1}(t-\tau)\right\}\right)^{2}\right)^{-1}\right) \\
&\left.\quad \times \quad \exp \left\{u_{2}(t-\tau)-u_{2}(t)\right\}\right] .
\end{aligned}
$$


Suppose $x=\left(u_{1}(t), u_{2}(t)\right)^{T} \in X$ is a solution of (24) and (25) for a certain $\lambda \in(0,1)$. Integrating (24), (25) over the interval $[0, \omega]$, we obtain

$$
\begin{aligned}
& \int_{0}^{\omega} a(t) \exp \left\{u_{1}(t)\right\} d t \\
& +\int_{0}^{\omega}\left(\left(4 G e \delta \exp \left\{u_{2}(t)\right\}+(4 G h-1) e^{2} \delta^{2}\right.\right. \\
& \left.\times \exp \left\{u_{1}(t)+u_{2}(t)\right\}\right) \\
& \left.\times\left(4 G\left(1+h e \delta \exp \left\{u_{1}(t)\right\}\right)^{2}\right)^{-1}\right) d t \\
& =\bar{r}_{1} \omega, \quad \\
& \int_{0}^{\omega} b_{1}(t-\tau) \exp \left\{-\int_{t-\tau}^{t} \beta(s) d s\right\} \\
& \times \exp \left\{u_{2}(t-\tau)-u_{2}(t)\right\} \\
& \times\left(\left(4 G e \delta B \exp \left\{u_{1}(t-\tau)\right\}+(4 G h-1) e^{2} \delta^{2} B\right.\right. \\
& \left.\times \exp \left\{2 u_{1}(t-\tau)\right\}\right) \\
& \left.\times\left(4 G\left(1+h e \delta \exp \left\{u_{1}(t-\tau)\right\}\right)^{2}\right)^{-1}\right) d t \\
& =\bar{r}_{2} \omega .
\end{aligned}
$$

It follows from $\left(A_{1}\right),(24)$, and (26) that

$$
\begin{gathered}
\int_{0}^{\omega}\left|\dot{u}_{1}(t)\right| d t \\
=\lambda \int_{0}^{\omega} \mid r_{1}(t)-a(t) \exp \left\{u_{1}(t)\right\} \\
-\left(\left(4 G e \delta \exp \left\{u_{2}(t)\right\}+(4 G h-1) e^{2} \delta^{2}\right.\right. \\
\left.\times \exp \left\{u_{1}(t)+u_{2}(t)\right\}\right) \\
\left.\times\left(4 G\left(1+h e \delta \exp \left\{u_{1}(t)\right\}\right)^{2}\right)^{-1}\right) \mid d t \\
<\int_{0}^{\omega} r_{1}(t) d t+\int_{0}^{\omega} a(t) \exp \left\{u_{1}(t)\right\} d t \\
+\int_{0}^{\omega}\left(\left(4 G e \delta \exp \left\{u_{2}(t)\right\}+(4 G h-1) e^{2} \delta^{2}\right.\right. \\
\left.\times \exp \left\{u_{1}(t)+u_{2}(t)\right\}\right) \\
\left.\times\left(4 G\left(1+h e \delta \exp \left\{u_{1}(t)\right\}\right)^{2}\right)^{-1}\right) d t \\
=\int_{0}^{\omega} r_{1}(t) d t+\bar{r}_{1} \omega=2 \bar{r}_{1} \omega ;
\end{gathered}
$$

that is,

$$
\int_{0}^{\omega}\left|\dot{u}_{1}(t)\right| d t<2 \bar{r}_{1} \omega
$$

Similarly, it follows from $\left(A_{1}\right),(25)$, and (27) that

$$
\int_{0}^{\omega}\left|\dot{u}_{2}(t)\right| d t<2 \bar{r}_{2} \omega .
$$

Since $\left(u_{1}(t), u_{2}(t)\right)^{T} \in X$, there exist $\xi_{i}, \eta_{i} \in[0, \omega]$ such that

$$
u_{i}\left(\xi_{i}\right)=\min _{t \in[0, \omega]} u_{i}(t), \quad u_{i}\left(\eta_{i}\right)=\max _{t \in[0, \omega]} u_{i}(t), \quad i=1,2
$$

Multiplying (25) by $\exp \left\{u_{2}(t)\right\}$ and integrating over $[0, \omega]$, we obtain

$$
\begin{aligned}
& \int_{0}^{\omega} r_{2}(t) \exp \left\{u_{2}(t)\right\} d t \\
& =\int_{0}^{\omega}\left[b_{1}(t-\tau) \exp \left\{-\int_{t-\tau}^{t} \beta(s) d s\right\}\right. \\
& \times\left(\left(4 G e \delta B \exp \left\{u_{1}(t-\tau)\right\}+(4 G h-1) e^{2} \delta^{2} B\right.\right. \\
& \left.\times \exp \left\{2 u_{1}(t-\tau)\right\}\right) \\
& \left.\times\left(4 G\left(1+h e \delta \exp \left\{u_{1}(t-\tau)\right\}\right)^{2}\right)^{-1}\right) \\
& \left.\times \exp \left\{u_{2}(t-\tau)\right\}\right] d t \\
& =\int_{-\tau}^{\omega-\tau}\left[b_{1}(\sigma) \exp \left\{-\int_{\sigma}^{\sigma+\tau} \beta(s) d s\right\}\right. \\
& \times\left(\left(4 G e \delta B \exp \left\{u_{1}(\sigma)\right\}+(4 G h-1) e^{2} \delta^{2} B\right.\right. \\
& \left.\times \exp \left\{2 u_{1}(\sigma)\right\}\right) \\
& \left.\times\left(4 G\left(1+h e \delta \exp \left\{u_{1}(\sigma)\right\}\right)^{2}\right)^{-1}\right) \\
& \left.\times \exp \left\{u_{2}(\sigma)\right\}\right] d t \\
& =\int_{0}^{\omega}\left[b_{1}(t) \exp \left\{-\int_{t}^{t+\tau} \beta(s) d s\right\}\right. \\
& \times\left(\left(4 G e \delta B \exp \left\{u_{1}(t)\right\}+(4 G h-1) e^{2} \delta^{2} B\right.\right. \\
& \left.\times \exp \left\{2 u_{1}(t)\right\}\right) \\
& \left.\times\left(4 G\left(1+h e \delta \exp \left\{u_{1}(t)\right\}\right)^{2}\right)^{-1}\right) \\
& \left.\times \exp \left\{u_{2}(t)\right\}\right] d t ;
\end{aligned}
$$


that is,

$$
\begin{aligned}
& \int_{0}^{\omega} r_{2}(t) \exp \left(u_{2}(t)\right) d t \\
&=\int_{0}^{\omega}\left[b_{1}(t) \exp \left\{-\int_{t}^{t+\tau} \beta(s) d s\right\}\right. \\
& \times\left(\left(4 G e \delta B \exp \left\{u_{1}(t)\right\}+(4 G h-1) e^{2} \delta^{2} B\right.\right. \\
&\left.\times \exp \left\{2 u_{1}(t)\right\}\right) \\
&\left.\times\left(4 G\left(1+h e \delta \exp \left\{u_{1}(t)\right\}\right)^{2}\right)^{-1}\right) \\
&\left.\times \exp \left\{u_{2}(t)\right\}\right] d t .
\end{aligned}
$$

It follows from (27), (33), and $\left(A_{1}\right)$; we see that

$$
\begin{aligned}
& r_{2}^{L} \int_{0}^{\omega} \exp \left\{u_{2}(t)\right\} d t \\
& \leq \int_{0}^{\omega} r_{2}(t) \exp \left\{u_{2}(t)\right\} d t \\
& =\int_{0}^{\omega}\left[b_{1}(t) \exp \left\{-\int_{t}^{t+\tau} \beta(s) d s\right\}\right. \\
& \times\left(\left(4 G e \delta B \exp \left\{u_{1}(t)\right\}+(4 G h-1) e^{2} \delta^{2} B\right.\right. \\
& \left.\times \exp \left\{2 u_{1}(t)\right\}\right) \\
& \left.\times\left(4 G\left(1+h e \delta \exp \left\{u_{1}(t)\right\}\right)^{2}\right)^{-1}\right) \\
& \left.\times \exp \left\{u_{2}(t)\right\}\right] d t \\
& \leq \int_{0}^{\omega} b_{1}(t) \exp \left\{-\int_{t}^{t+\tau} \beta(s) d s\right\} \\
& \times\left[\frac{4 G e \delta B \exp \left\{u_{1}(t)\right\}}{4 G\left(1+h e \delta \exp \left\{u_{1}(t)\right\}\right)^{2}}\right. \\
& \left.+\frac{(4 G h-1) e^{2} \delta^{2} B \exp \left\{2 u_{1}(t)\right\}}{4 G h^{2} e^{2} \delta^{2} \exp \left\{2 u_{1}(t)\right\}}\right] \\
& \times \exp \left\{u_{2}(t)\right\} d t \\
& \leq b_{1}^{M} \exp \left\{-\tau \beta^{L}\right\} \\
& \times\left[\frac{e \delta B \exp \left\{u_{1}\left(\eta_{1}\right)\right\}}{\left(1+h e \delta \exp \left\{u_{1}\left(\xi_{1}\right)\right\}\right)^{2}}\right. \\
& \left.+\frac{(4 G h-1) B}{4 G h^{2}}\right] \int_{0}^{\omega} \exp \left\{u_{2}(t)\right\} d t
\end{aligned}
$$

which implies

$$
\begin{aligned}
r_{2}^{L} \leq & b_{1}^{M} \exp \left\{-\tau \beta^{L}\right\} \\
& \times\left[\frac{e \delta B \exp \left\{u_{1}\left(\eta_{1}\right)\right\}}{\left(1+h e \delta \exp \left\{u_{1}\left(\xi_{1}\right)\right\}\right)^{2}}+\frac{(4 G h-1) B}{4 G h^{2}}\right] .
\end{aligned}
$$

So

$$
\begin{aligned}
u_{1}\left(\eta_{1}\right) \geq \ln ( & \left(\left[4 G h^{2} r_{2}^{L}\left(b_{1}^{M}\right)^{-1} \exp \left\{\tau \beta^{L}\right\}-(4 G h-1) B\right]\right. \\
& \left.\times\left(1+h e \delta \exp \left\{u_{1}\left(\xi_{1}\right)\right\}\right)^{2}\right) \\
& \left.\times\left(4 G h^{2} e \delta B\right)^{-1}\right) .
\end{aligned}
$$

This, combined with (29), gives

$$
\begin{aligned}
u_{1}(t) \geq u_{1}\left(\eta_{1}\right) & -\int_{0}^{\omega}\left|\dot{u}_{1}(t)\right| d t \\
> & \ln \left(\left(\left[4 G h^{2} r_{2}^{L}\left(b_{1}^{M}\right)^{-1} \exp \left\{\tau \beta^{L}\right\}-(4 G h-1) B\right]\right.\right. \\
& \left.\times\left(1+h e \delta \exp \left\{u_{1}\left(\xi_{1}\right)\right\}\right)^{2}\right) \\
& \left.\times\left(4 G h^{2} e \delta B\right)^{-1}\right)-2 \bar{r}_{1} \omega .
\end{aligned}
$$

In particular, we have

$$
\begin{aligned}
u_{1}\left(\xi_{1}\right)>\ln (([ & \left.4 G h^{2} r_{2}^{L}\left(b_{1}^{M}\right)^{-1} \exp \left\{\tau \beta^{L}\right\}-(4 G h-1) B\right] \\
& \left.\times\left(1+h e \delta \exp \left\{u_{1}\left(\xi_{1}\right)\right\}\right)^{2}\right) \\
& \left.\times\left(4 G h^{2} e \delta B\right)^{-1}\right)-2 \bar{r}_{1} \omega
\end{aligned}
$$

or

$$
\begin{aligned}
& {\left[4 G h^{2} r_{2}^{L}\left(b_{1}^{M}\right)^{-1} \exp \left\{\tau \beta^{L}\right\}-(4 G h-1) B\right] h^{2} e^{2} \delta^{2}} \\
& \quad \times \exp \left\{2 u_{1}\left(\xi_{1}\right)\right\} \\
& \quad-\left[4 G h^{2} e \delta B \exp \{2 \bar{r} \omega\}\right. \\
& \left.\quad-2 h e \delta\left(4 G h^{2} r_{2}^{L}\left(b_{1}^{M}\right)^{-1} \exp \left\{\tau \beta^{L}\right\}-(4 G h-1) B\right)\right] \\
& \quad \times \exp \left\{u_{1}\left(\xi_{1}\right)\right\} \\
& +\left[4 G h^{2} r_{2}^{L}\left(b_{1}^{M}\right)^{-1} \exp \left\{\tau \beta^{L}\right\}-(4 G h-1) B\right]<0 .
\end{aligned}
$$

In view of $\left(A_{2}\right)$, we have

$$
\ln l_{-}<u_{1}\left(\xi_{1}\right)<\ln l_{+} .
$$


Similarly, it follows from (33) that

$$
\begin{aligned}
& b_{1}^{L} \exp \left\{-\tau \beta^{M}\right\} \\
& \times \frac{4 G e \delta B \exp \left\{u_{1}\left(\xi_{1}\right)\right\}+(4 G h-1) e^{2} \delta^{2} B \exp \left\{2 u_{1}\left(\xi_{1}\right)\right\}}{4 G\left(1+h e \delta \exp \left\{u_{1}\left(\eta_{1}\right)\right\}\right)^{2}} \\
& \times \int_{0}^{\omega} \exp \left\{u_{2}(t)\right\} d t \\
& \leq \int_{0}^{\omega} b_{1}(t) \exp \left\{-\int_{t}^{t+\tau} \beta(s) d s\right\} \\
& \quad \times \frac{4 G e \delta B \exp \left\{u_{1}(t)\right\}+(4 G h-1) e^{2} \delta^{2} B \exp \left\{2 u_{1}(t)\right\}}{4 G\left(1+h e \delta \exp \left\{u_{1}(t)\right\}\right)^{2}} \\
& \quad \times \exp \left\{u_{2}(t)\right\} d t \\
& =\int_{0}^{\omega} r_{2}(t) \exp \left\{u_{2}(t)\right\} d t \\
& \leq r_{2}^{M} \int_{0}^{\omega} \exp \left\{u_{2}(t)\right\} d t
\end{aligned}
$$

which implies

$$
\begin{aligned}
& b_{1}^{L} \exp \left\{-\tau \beta^{M}\right\} \\
& \times \frac{4 G e \delta B \exp \left\{u_{1}\left(\xi_{1}\right)\right\}+(4 G h-1) e^{2} \delta^{2} B \exp \left\{2 u_{1}\left(\xi_{1}\right)\right\}}{4 G\left(1+h e \delta \exp \left\{u_{1}\left(\eta_{1}\right)\right\}\right)^{2}} \\
& \leq r_{2}^{M}
\end{aligned}
$$

that is,

$$
b_{1}^{L} \exp \left\{-\tau \beta^{M}\right\} d s \frac{e \delta B \exp \left\{u_{1}\left(\xi_{1}\right)\right\}}{\left(1+h e \delta \exp \left\{u_{1}\left(\eta_{1}\right)\right\}\right)^{2}} \leq r_{2}^{M} .
$$

So

$$
u_{1}\left(\xi_{1}\right) \leq \ln \frac{r_{2}^{M}\left(1+h e \delta \exp \left\{u_{1}\left(\eta_{1}\right)\right\}\right)^{2}}{b_{1}^{L} e \delta B \exp \left\{-\tau \beta^{M}\right\}} .
$$

This, combined with (29), gives

$$
\begin{aligned}
u_{1}(t) \leq & u_{1}\left(\xi_{1}\right)+\int_{0}^{\omega}\left|\dot{u}_{1}(t)\right| d t \\
< & \ln \frac{r_{2}^{M}\left(1+h e \delta \exp \left\{u_{1}\left(\eta_{1}\right)\right\}\right)^{2}}{b_{1}^{L} e \delta B \exp \left\{-\tau \beta^{M}\right\}} \\
& +2 \bar{r}_{1} \omega .
\end{aligned}
$$

In particular, we have

$$
\begin{aligned}
u_{1}\left(\eta_{1}\right)< & \ln \frac{r_{2}^{M}\left(1+h e \delta \exp \left\{u_{1}\left(\eta_{1}\right)\right\}\right)^{2}}{b_{1}^{L} e \delta B \exp \left\{-\tau \beta^{M}\right\}} \\
& +2 \bar{r}_{1} \omega,
\end{aligned}
$$

or

$$
\begin{aligned}
& r_{2}^{M} h^{2} e^{2} \delta^{2} \exp \left\{2 u_{1}\left(\eta_{1}\right)\right\} \\
& \quad-\left[b_{1}^{L} e \delta B \exp \left\{-\tau \beta^{M}\right\} \exp \left\{-2 \bar{r}_{1} \omega\right\}-2 h e \delta r_{2}^{M}\right] \\
& \quad \times \exp \left\{u_{1}\left(\eta_{1}\right)\right\}+r_{2}^{M}>0
\end{aligned}
$$

It follows from $\left(A_{2}\right)$ that

$$
u_{1}\left(\eta_{1}\right)<\ln h_{-} \quad \text { or } \quad u_{1}\left(\eta_{1}\right)>\ln h_{+} .
$$

From (29) and (40), we find

$$
\begin{aligned}
u_{1}(t) & \leq u_{1}\left(\xi_{1}\right)+\int_{0}^{\omega}\left|\dot{u}_{1}(t)\right| d t \\
& <\ln l_{+}+2 \bar{r}_{1} \omega \triangleq H_{11} .
\end{aligned}
$$

On the other hand, it follows from $\left(A_{1}\right),(26)$, and (49) that

$$
\begin{aligned}
\bar{r}_{1} \omega \geq & \int_{0}^{\omega} \frac{4 G e \delta \exp \left\{u_{2}\left(\xi_{2}\right)\right\}}{4 G\left(1+h e \delta \exp \left\{\ln l_{+}+2 \bar{r} \omega\right\}\right)^{2}} d t \\
\bar{r}_{1} \omega \leq & \int_{0}^{\omega} a(t) \exp \left\{\ln l_{+}+2 \bar{r}_{1} \omega\right\} d t \\
& +\int_{0}^{\omega} e \delta \exp \left\{u_{2}\left(\eta_{2}\right)\right\} d t \\
& +\int_{0}^{\omega} \frac{e \delta \exp \left\{u_{2}\left(\eta_{2}\right)\right\}}{2} d t .
\end{aligned}
$$

It follows from (50) that

$$
u_{2}\left(\xi_{2}\right) \leq \ln \frac{\bar{r}_{1}\left(1+h e \delta \exp \left\{\ln l_{+}+2 \bar{r}_{1} \omega\right\}\right)^{2}}{e \delta} .
$$

This, combined with (30), gives

$$
\begin{aligned}
u_{2}(t) \leq & u_{2}\left(\xi_{2}\right)+\int_{0}^{\omega}\left|\dot{u}_{2}(t)\right| d t \\
< & \ln \frac{\bar{r}_{1}\left(1+h e \delta \exp \left\{\ln l_{+}+2 \bar{r}_{1} \omega\right\}\right)^{2}}{e \delta} \\
& +2 \bar{r}_{2} \omega \triangleq H_{21} .
\end{aligned}
$$

Moreover, because of $\left(A_{3}\right)$, it follows from (51) that

$$
u_{2}\left(\eta_{2}\right) \geq \ln \frac{2\left(\bar{r}_{1}-\bar{a} \exp \left\{\ln l_{+}+2 \bar{r} \omega\right\}\right)}{3 e \delta} .
$$

This, combined with (30) again, gives

$$
\begin{aligned}
u_{2}(t) \geq & u_{2}\left(\eta_{2}\right)-\int_{0}^{\omega}\left|\dot{u}_{2}(t)\right| d t \\
> & \ln \frac{2\left(\bar{r}_{1}-\bar{a} \exp \left\{\ln l_{+}+2 \bar{r} \omega\right\}\right)}{3 e \delta} \\
& -2 \bar{r}_{2} \omega \triangleq H_{22} .
\end{aligned}
$$


It follows from (53) and (55) that

$$
\max _{t \in[0, \omega]} u_{2}(t)<\max \left\{\left|H_{21}\right|,\left|H_{22}\right|\right\} \triangleq H_{2}
$$

Now, let us consider QNx with

$$
\begin{aligned}
Q N & \left(u_{1}, u_{2}\right)^{T} \\
= & \left(\bar{r}_{1} \omega-\bar{a} \omega \exp \left\{u_{1}\right\}-w\right. \\
& \times \frac{4 G e \delta \exp \left\{u_{2}\right\}+(4 G h-1) e^{2} \delta^{2} \exp \left\{u_{1}+u_{2}\right\}}{4 G\left(1+h e \delta \exp \left\{u_{1}\right\}\right)^{2}}, \\
& -\bar{r}_{2} \omega+\bar{b} \omega \\
& \left.\times \frac{4 G e \delta B \exp \left\{u_{1}\right\}+(4 G h-1) e^{2} \delta^{2} B \exp \left\{2 u_{1}\right\}}{4 G\left(1+h e \delta \exp \left\{u_{1}\right\}\right)^{2}}\right)^{T} .
\end{aligned}
$$

In view of $\left(A_{1}\right),\left(A_{2}\right)$, and $\left(A_{3}\right)$, we can show that the equation $\mathrm{QN}\left(u_{1}, u_{2}\right)^{T}=0$ has two distinct solutions

$$
\begin{aligned}
& \tilde{u}=\left(\ln u_{-}, \ln \frac{4 G\left(\bar{r}_{1}-\bar{a} u_{-}\right)\left(1+h e \delta u_{-}\right)^{2}}{4 G e \delta+(4 G h-1) e^{2} \delta^{2} u_{-}}\right), \\
& \widehat{u}=\left(\ln u_{+}, \ln \frac{4 G\left(\bar{r}_{1}-\bar{a} u_{+}\right)\left(1+h e \delta u_{+}\right)^{2}}{4 G e \delta+(4 G h-1) e^{2} \delta^{2} u_{+}}\right) .
\end{aligned}
$$

Choose $C>0$ such that

$$
\begin{gathered}
C>\max \left\{\left|\ln \frac{4 G\left(\bar{r}_{1}-\bar{a} u_{-}\right)\left(1+h e \delta u_{-}\right)^{2}}{4 G e \delta+(4 G h-1) e^{2} \delta^{2} u_{-}}\right|,\right. \\
\left.\left|\ln \frac{4 G\left(\bar{r}_{1}-\bar{a} u_{+}\right)\left(1+h e \delta u_{+}\right)^{2}}{4 G e \delta+(4 G h-1) e^{2} \delta^{2} u_{+}}\right|\right\} .
\end{gathered}
$$

We define two open bounded subsets. Let

$$
\begin{aligned}
& \Omega_{1}=\left\{x=\left(u_{1}, u_{2}\right)^{T} \in X \mid u_{1}(t) \in\left(\ln l_{-}, \ln h_{-}\right),\right. \\
& \left.\max _{t \in[0, \omega]}\left|u_{2}(t)\right|<H_{2}+C\right\}, \\
& \Omega_{2}=\left\{x=\left(u_{1}, u_{2}\right)^{T} \in X \mid \min _{t \in[0, \omega]} u_{1}(t) \in\left(\ln l_{-}, \ln l_{+}\right),\right. \\
& \left.\max _{t \in[0, \omega]} u_{1}(t) \in\left(\ln h_{+}, H_{11}\right), \max _{t \in[0, \omega]}\left|u_{2}(t)\right|<H_{2}+C\right\} .
\end{aligned}
$$

Then both $\Omega_{1}$ and $\Omega_{2}$ are bounded open subsets of $X$. It follows from (16) and (59) that $\tilde{u} \in \Omega_{1}$ and $\widehat{u} \in \Omega_{2}$. With the help of (16), (40), (48), (49), (56), and (59), it is easy to see that $\Omega_{1} \cap \Omega_{2}=\phi$, and $\Omega_{i}$ satisfies the requirement (a) in Lemma 3 for $i=1,2$. Moreover, $Q N x \neq 0$ for $x \in \partial \Omega \cap \operatorname{Ker} L=\partial \Omega \cap \mathbb{R}^{2}$. A direct computation gives $\operatorname{deg}\left\{J Q N, \Omega_{i} \cap \operatorname{Ker} L, 0\right\} \neq 0$. Here,
$J$ is taken as the identity mapping since $\operatorname{Im} Q=\operatorname{Ker} L$. So far we have proved that $\Omega_{i}$ satisfies all the assumptions in Lemma 3. Hence, (16) has at least two $\omega$-periodic solutions $u^{*}(t)$ and $u^{+}(t)$ with $u^{*} \in \operatorname{Dom} L \cap \bar{\Omega}_{1}$ and $u^{+} \in \operatorname{Dom} L \cap \bar{\Omega}_{2}$. Obviously, $u^{*}$ and $u^{+}$are different. Let $x^{*}(t)=\exp \left(u_{1}^{*}(t)\right)$, $y_{2}^{*}(t)=\exp \left(u_{2}^{*}(t)\right)$ and $x^{+}(t)=\exp \left(u_{1}^{+}(t)\right), y_{2}^{+}(t)=$ $\exp \left(u_{2}^{+}(t)\right)$. Then, by (18), $\left(x^{*}(t), y_{2}^{*}(t)\right)$ and $\left(x^{+}(t), y_{2}^{+}(t)\right)$ are two different positive $\omega$-periodic solutions of $(4)$. By the periodicity of the coefficients of system (4), it is not difficult to verify that

$$
\begin{aligned}
g^{*}(t)=- & \beta(t) y_{1}(t) \\
+ & \left(\left(b_{1}(t) 4 G e \delta B x^{*}(t) y_{2}^{*}(t)\right.\right. \\
& \left.+(4 G h-1) e^{2} \delta^{2} B\left(x^{*}\right)^{2}(t) y_{2}^{*}(t)\right) \\
& \left.\times\left(4 G\left(1+h e \delta x^{*}(t)\right)^{2}\right)^{-1}\right) \\
- & b_{1}(t-\tau) \exp \left(-\int_{t-\tau}^{t} \beta(s) d s\right) \\
\times & \left(4 G e \delta B x^{*}(t-\tau) y_{2}^{*}(t-\tau)\right. \\
& \left.+(4 G h-1) e^{2} \delta^{2} B\left(x^{*}\right)^{2}(t-\tau) y_{2}^{*}(t-\tau)\right) \\
& \left.\times\left(4 G\left(1+h e \delta x^{*}(t-\tau)\right)^{2}\right)^{-1}\right)
\end{aligned}
$$

is also $\omega$-periodic. Then, from Lemma 4 , we know that

$$
\frac{d y_{1}(t)}{d t}=-\beta(t) y_{1}(t)+g^{*}(t)
$$

has a unique $\omega$-periodic solution denoted by $y_{1}^{*}(t)$. And

$$
\frac{d y_{2}(t)}{d t}=-\beta(t) y_{1}(t)+g^{+}(t)
$$

has a unique $\omega$-periodic solution denoted by $y_{1}^{+}(t)$. Therefore, $\left(x^{*}(t), y_{1}^{*}(t), y_{2}^{*}(t)\right)$ and $\left(x^{+}(t), y_{1}^{+}(t), y_{2}^{+}(t)\right)$ are two different $\omega$-periodic solutions of system (3). This completes the proof of Theorem 5 .

\section{Conflict of Interests}

The authors declare that there is no conflict of interests regarding the publication of this paper.

\section{Funding}

This work is supported by JB12254.

\section{References}

[1] Y. L. Song, Y. Peng, and J. J. Wei, "Bifurcations for a predatorprey system with two delays," Journal of Mathematical Analysis and Applications, vol. 337, no. 1, pp. 466-479, 2008. 
[2] S. L. Yuan and Y. L. Song, "Stability and Hopf bifurcations in a delayed Leslie-Gower predator-prey system," Journal of Mathematical Analysis and Applications, vol. 355, no. 1, pp. 82$100,2009$.

[3] Y. L. Song and S. L. Yuan, "Bifurcation analysis for a regulated logistic growth model," Applied Mathematical Modelling, vol. 31, no. 9, pp. 1729-1738, 2007.

[4] Y. H. Xia and M. Han, "New conditions on the existence and stability of periodic solution in Lotka-Volterra's population system," SIAM Journal on Applied Mathematics, vol. 69, no. 6, pp. 1580-1597, 2009.

[5] T. Zhang, J. Liu, and Z. Teng, "Existence of positive periodic solutions of an SEIR model with periodic coefficients," Applications of Mathematics, vol. 57, no. 6, pp. 601-616, 2012.

[6] Y. H. Xia, "Global asymptotic stability of an almost periodic nonlinear ecological model," Communications in Nonlinear Science and Numerical Simulation, vol. 16, no. 11, pp. 4451-4478, 2011.

[7] Y. H. Xia, "Global analysis of an impulsive delayed LotkaVolterra competition system," Communications in Nonlinear Science and Numerical Simulation, vol. 16, no. 3, pp. 1597-1616, 2011.

[8] Y. Xia, X. Yuan, K. I. Kou, and P. J. Y. Wong, "Existence and uniqueness of solution for perturbed nonautonomous systems with nonuniform exponential dichotomy," Abstract and Applied Analysis, vol. 2014, Article ID 725098, 10 pages.

[9] Y. H. Xia, "Periodic solution of certain nonlinear differential equations: via topological degree theory and matrix spectral theory," International Journal of Bifurcation and Chaos in Applied Sciences and Engineering, vol. 22, no. 8, Article ID 1250196, 17 pages, 2012.

[10] L. Yin and Z. Zhang, "Existence of a positive solution for a firstorder p-Laplacian BVP with impulsive on time scales," Journal of Applied Analysis and Computation, vol. 2, no. 1, pp. 103-109, 2012.

[11] X. Gu, H. Wang, P. Wong, and Y. H. Xia, "Existence and stability of periodic solution to delayed nonlinear differential equations," Abstract and Applied Analysis, vol. 2014, Article ID 156948, 12 pages, 2014.

[12] W. Ding and M. Han, "Dynamic of a non-autonomous predatorprey system with infinite delay and diffusion," Computers \& Mathematics with Applications, vol. 56, no. 5, pp. 1335-1350, 2008.

[13] Y. F. Gao, P. J. Y. Wong, Y. H. Xia, and X. Yuan, "Multiple periodic solutions of a nonautonomous plant-hare model," Abstract and Applied Analysis, vol. 2014, Article ID 130856, 7 pages, 2014.

[14] W. G. Aiello and H. I. Freedman, "A time-delay model of singlespecies growth with stage structure," Mathematical Biosciences, vol. 101, no. 2, pp. 139-153, 1990.

[15] W. Wang and L. Chen, "A predator-prey system with stagestructure for predator," Computers \& Mathematics with Applications, vol. 33, no. 8, pp. 83-91, 1997.

[16] Y. H. Xia, J. Cao, and S. S. Cheng, "Multiple periodic solutions of a delayed stage-structured predator-prey model with nonmonotone functional responses," Applied Mathematical Modelling, vol. 31, no. 9, pp. 1947-1959, 2007.

[17] R. E. Gaines and J. L. Mawhin, Coincidence Degree, and Nonlinear Differential Equations, Lecture Notes in Mathematics, Springer, Berlin, Germany, 1977. 


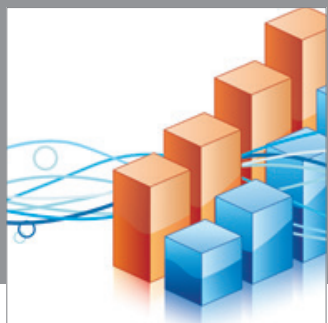

Advances in

Operations Research

mansans

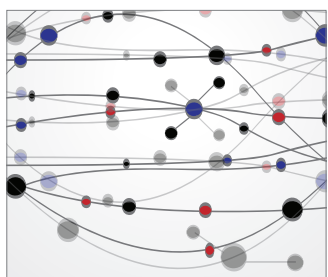

The Scientific World Journal
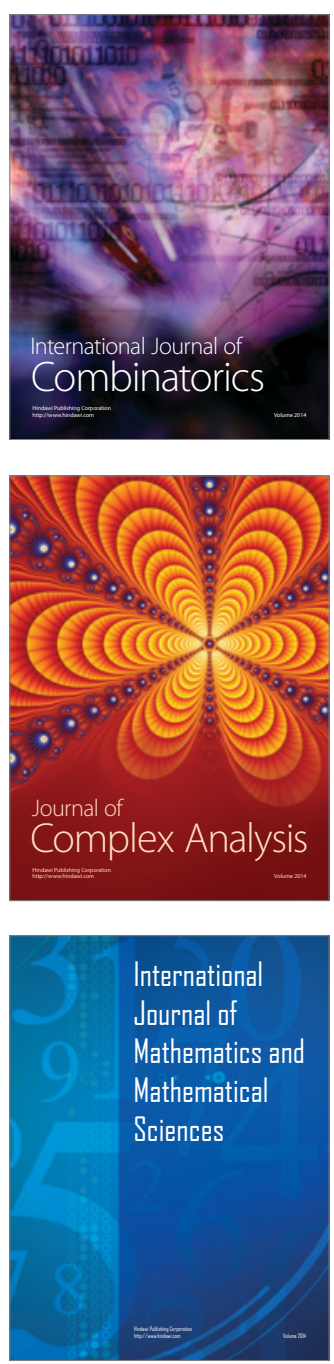
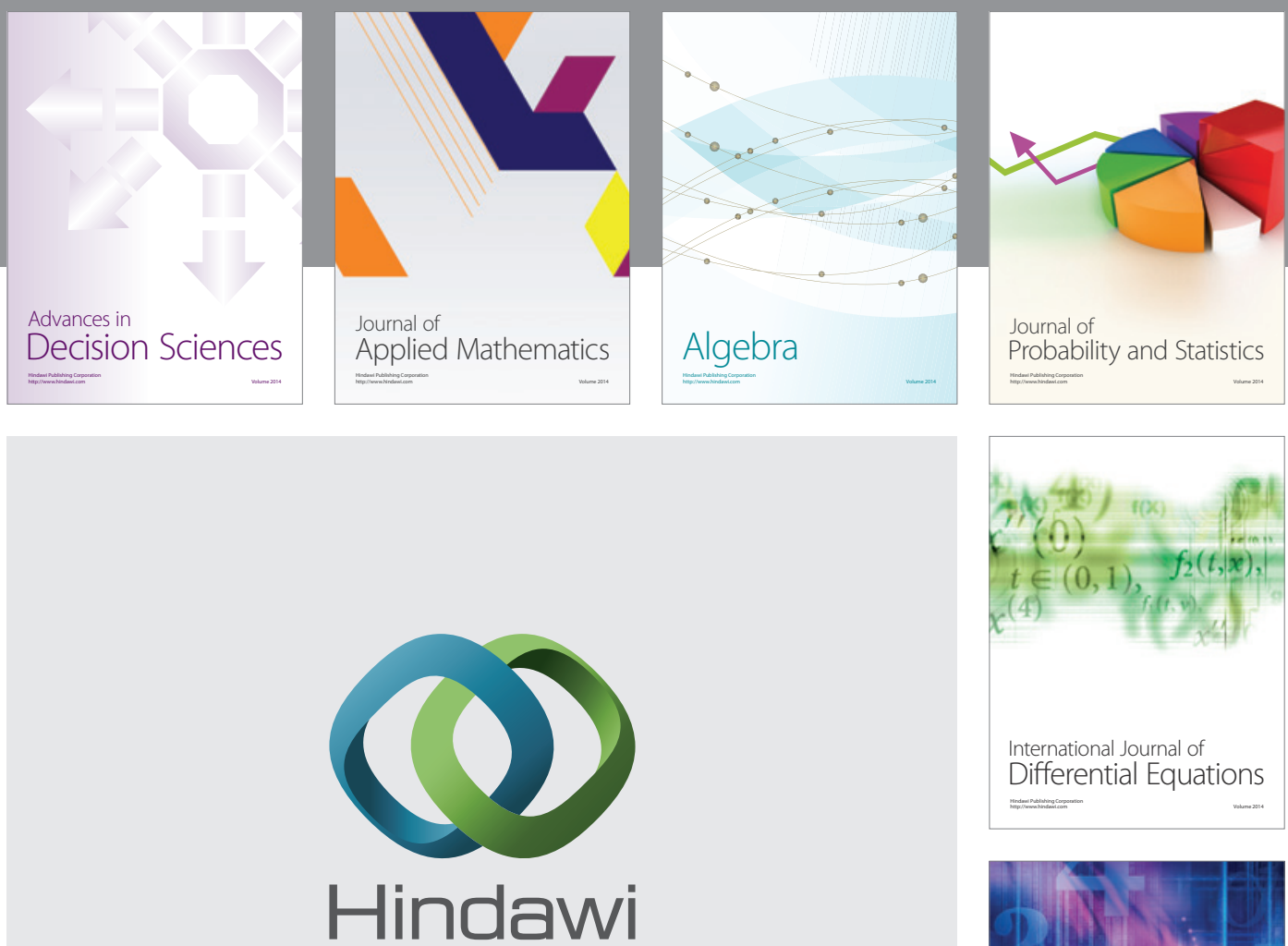

Submit your manuscripts at http://www.hindawi.com
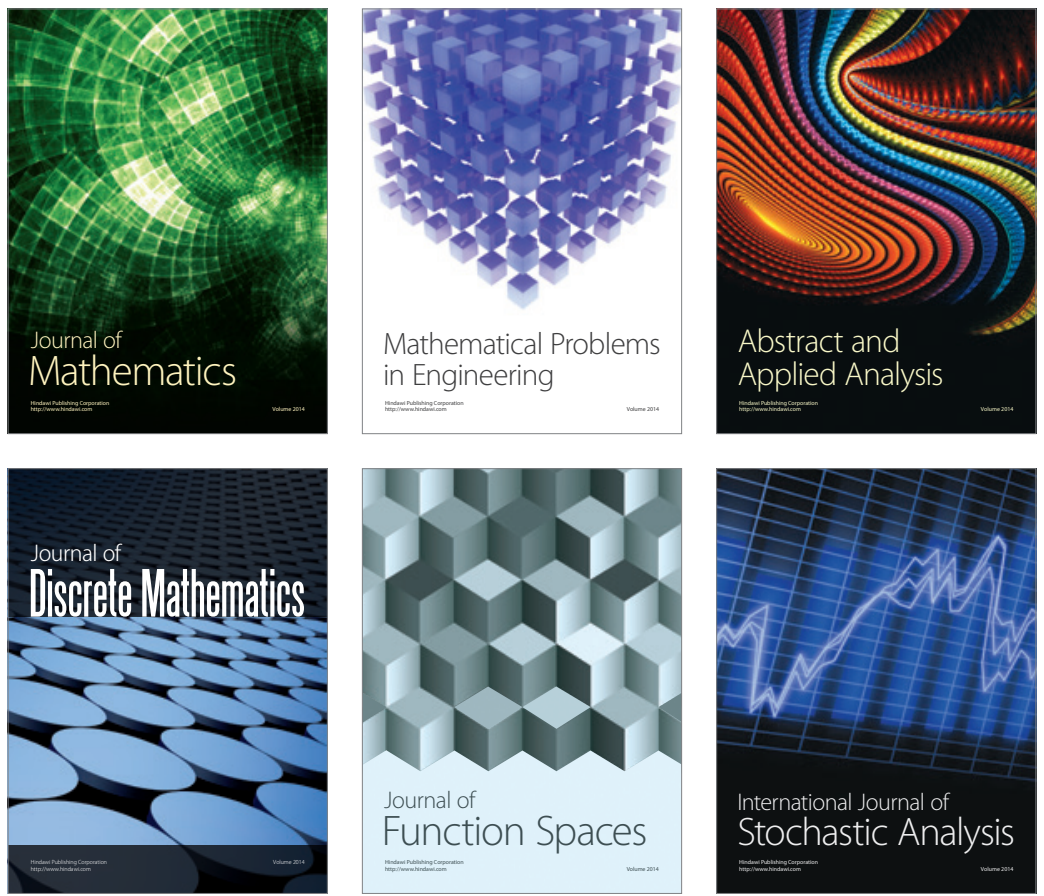

Journal of

Function Spaces

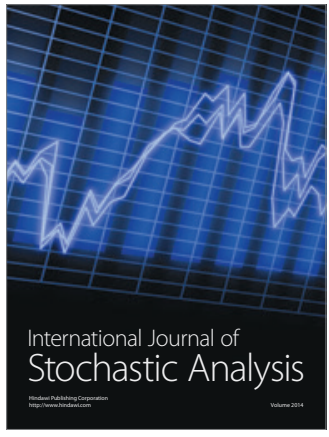

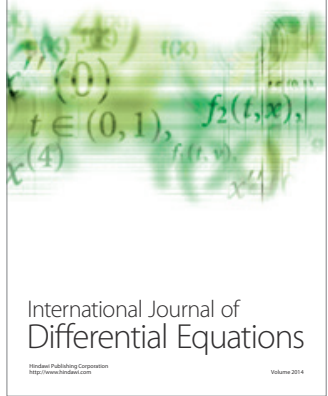
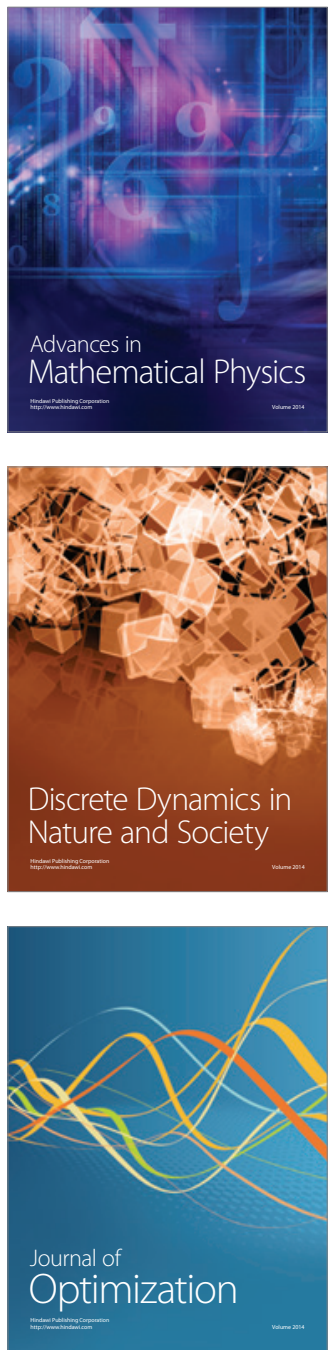